Research Article

\title{
Propagation and Attenuation of Elastic Waves in a Double Porosity Medium
}

\author{
J. S. Nandal ${ }^{1}$ and T. N. Saini ${ }^{2}$ \\ ${ }^{1}$ Department of Mathematics, M.D. University, Rohtak 124001, India \\ ${ }^{2}$ Department of Mathematics, Government College Kalka, Kalka 133302, India
}

Correspondence should be addressed to T. N. Saini; tns.kalka@gmail.com

Received 5 December 2012; Accepted 26 December 2012

Academic Editors: G. Casula, H. Perroud, and A. Streltsov

Copyright (C) 2013 J. S. Nandal and T. N. Saini. This is an open access article distributed under the Creative Commons Attribution License, which permits unrestricted use, distribution, and reproduction in any medium, provided the original work is properly cited.

\begin{abstract}
This study solves the mathematical model for the propagation of harmonic plane waves in a dissipative double porosity solid saturated by a viscous fluid. The existence of three dilatational waves is explained through three scalar potentials satisfying wave equations. Velocities of these waves are obtained from the roots of a cubic equation. Lone shear wave is identified through a vector potential satisfying a wave equation. The displacements of solid particles are expressed through these four potentials. The displacements of fluid particles in pores and fractures can also be expressed in terms of these potentials. A numerical example is solved to calculate the complex velocities of four waves in a dissipative double porosity solid. Each of the complex velocities is resolved to define the phase velocity and quality factor of attenuation for the corresponding wave. Effects of medium properties and wave frequency are analyzed numerically on the propagation characteristics of four attenuated waves. It seems that $P_{1}$ and $S$ waves are not very sensitive to the pore/fluids characteristics, except the fracture porosity. Hence, the recovery and analysis of slower $\left(P_{2}\right.$, $P_{3}$ ) waves become more desired to understand the fluid-rock dynamism in crustal rocks.
\end{abstract}

\section{Introduction}

Pores are pervasive in most of the igneous, metamorphic, and sedimentary rocks in the earth's crust. Traditional approaches to seismic exploration often make use of Biot's theory of poroelasticity. This theory has always been limited by an explicit assumption that the porosity itself is homogeneous. For acoustic analysis of many rock samples in a laboratory setting, this assumption is known to be adequate. But, in the modeling of real heterogeneous reservoirs, it may not be a realistic assumption. In fact, porosity found in the earth may have many shapes and sizes, but two types of porosity are more important. One is matrix (or storage) porosity that occupies a finite and substantial fraction of the volume of a porous rock. Other is fracture or crack porosity that may occupy very little volume, but fluid flow occurs primarily through the fracture network. However, fluid storage occurs mostly in the porous matrix.

This model identified as double porosity model $[1,2]$ considers a fracture network that divides the porous matrix into different blocks and the fluid in fractures surrounds the disaggregated matrix blocks supported entirely by fluid pressure. In fact, most of the near-surface rock masses are fractured to some degree. It demands to examine the coupled fluid-rock deformation through the double porosity network by extending the Biot's theory [3-5] to the composite containing fracture network in porous matrix. There are some attempts, recorded in the literature, in which fractures are incorporated into the rock models. These attempts account for the partial saturation effects and the fluid-flow during the passage of seismic waves [6-11]. In these attempts, the approach has been limited mainly to modify the elastic parameters of Biot's theory for the introduction of cracks.

The previous double porosity models are proposed by Wilson and Aifantis [12, 13], Cho et al. [14], and Bai et al. [15]. These were based on the mixture of solid and fluid phases. That means, coupling between fluid flow and solid deformations is ignored. Hence, to consider this coupling, it becomes necessary to incorporate Biot's concepts of poroelasticity into the double porosity model. Berryman and 
Wang $[16,17]$ made efforts for a rigorous extension of Biot's poroelasticity to include fractures/cracks by making a generalization to double-porosity/dual-permeability modeling. They derived the phenomenological equations and presented the method to determine the relevant coefficients. Their discussions showed that three compressional waves in double porosity media are diffusive. Based on the volume averaging technology, Pride and Berryman $[18,19]$ derived the governing equations of fluid-saturated double porosity media. In addition, the fluid transport mechanism was also investigated and a symmetric dual-permeability Darcy law was established.

Viscoelasticity is a widely accepted property of many rocks in the crust and is a major cause of seismic attenuation. Moreover, in the presence of double porosity, a viscoelastic solid permeated by pores and fractures and saturated with viscous fluid represents a much realistic model for sedimentary or reservoir rocks. The present work considers the propagation of attenuated waves in this dissipative poroviscoelastic composite medium. Three scalar potentials identify three dilatational waves and a vector potential identifies the lone shear wave in the considered porous medium. Complex velocities associated to these four attenuated waves are resolved to define their propagation velocities as well as attenuation coefficients. Effects of frequency, frame anelasticity, pore-fluid viscosity, porosity, and fracture permeability are observed on the phase velocities and attenuation coefficients of the four waves.

\section{Basic Equations}

The double porosity medium consists of three constituents, that is, solid matrix, pore fluid, and fracture fluid, which are identified with indices ' $s$, ' $p$ ', ' $c$ ', respectively. In this porous aggregate, volume fractions of the fluid in pores $\left(\epsilon_{p}\right)$ and in fractures $\left(\epsilon_{c}\right)$ define the total porosity $f\left(=\epsilon_{p}+\epsilon_{c}\right)$ of solid skeletal and then $\epsilon_{s}(=1-f)$ is the volume fraction for solid grains. Following Berryman and Wang [16, 17], the equations of motion for low-frequency vibrations of constituent particles in double porosity media, in the absence of body forces, are given by

$$
\begin{gathered}
\tau_{i j, j}=\epsilon_{s} \rho_{s} \ddot{u}_{i}+\rho_{12} \ddot{v}_{i}+\rho_{13} \ddot{w}_{i}-b_{12} \dot{v}_{i}-b_{13} \dot{w}_{i}, \\
-\epsilon_{p}\left(P_{p}\right)_{, i}=\epsilon_{p} \rho_{f} \ddot{u}_{i}+\rho_{22} \ddot{v}_{i}+\rho_{23} \ddot{w}_{i}+b_{12} \dot{v}_{i}+b_{23}\left(\dot{v}_{i}-\dot{w}_{i}\right), \\
-\epsilon_{c}\left(P_{c}\right)_{, i}=\epsilon_{c} \rho_{f} \ddot{u}_{i}+\rho_{23} \ddot{v}_{i}+\rho_{33} \ddot{w}_{i}+b_{13} \dot{w}_{i}-b_{23}\left(\dot{v}_{i}-\dot{w}_{i}\right),
\end{gathered}
$$

where $\tau_{i j}$ is the stress tensor for saturated (undrained) porous solid and $P_{p}, P_{c}$ are fluid pressures in pore space and fracture space, respectively. $\rho_{s}$ and $\rho_{f}$ are intrinsic densities of solid grains and pore fluid, respectively. $u_{i}$ are the components of displacement $(\mathbf{u})$ of solid particles. The components $v_{i}$ and $w_{i}$ denote displacements ( $\mathbf{v}$ and $\mathbf{w}$ ) in pore fluid and fracture fluid relative to solid frame, respectively. English indices (other than $s, p, c$ ) take values 1,2, and 3. Repetition of any of these indices implies summation. Dot over a variable implies partial derivative with time and comma before an index implies partial space differentiation. Dynamical constants and dissipation parameters in (1) are defined as follows:

$$
\begin{gathered}
\rho_{12}=\frac{\left[\epsilon_{c}\left(\tau_{c}-1\right)-\epsilon_{p}\left(\tau_{p}-1\right)-f(\tau-1)\right] \rho_{f}}{2}, \\
\rho_{13}=\frac{\left[\epsilon_{p}\left(\tau_{p}-1\right)-\epsilon_{c}\left(\tau_{c}-1\right)-f(\tau-1)\right] \rho_{f}}{2}, \\
\rho_{23}=\frac{\left[f(\tau-1)-\epsilon_{p}\left(\tau_{p}-1\right)-\epsilon_{c}\left(\tau_{c}-1\right)\right] \rho_{f}}{2}, \\
\rho_{33}=\epsilon_{c} \tau_{c} \rho_{f} ; \\
b_{12}=\frac{\rho_{f} \epsilon_{p}\left(\epsilon_{p} \chi_{22}-\epsilon_{c} \chi_{12}\right) \eta}{\chi_{0}} \\
\chi_{0}=\chi_{11} \chi_{22}-\chi_{12}^{2}, \\
b_{13}=\frac{\rho_{f} \epsilon_{c}\left(\epsilon_{c} \chi_{11}-\epsilon_{p} \chi_{12}\right) \eta}{\chi_{0}} \\
b_{23}=\frac{\rho_{f} \epsilon_{p} \epsilon_{c} \chi_{12} \eta}{\chi_{0}},
\end{gathered}
$$

where $\eta$ is shear (kinematic) viscosity of interstitial fluid and the tensor components $\chi_{i j}$ define permeability of solid frame. Tortuosity parameters relate to shape factor $r(=$ 0.5 for spherical grains) and volume fractions as $\tau=1+$ $r \epsilon_{s} / f, \tau_{p}=1+r \epsilon_{s} / \epsilon_{p}$, and $\tau_{c}=1$.

Following Berryman and Wang $[16,17]$, constitutive relations for stresses in porous frame and hydrostatic pressures in pore fluid and fracture fluid are given by

$$
\begin{gathered}
\tau_{i j}=K_{u}\left(\nabla \cdot \mathbf{u}-B^{p} \epsilon_{p} \nabla \cdot \mathbf{v}-B^{c} \epsilon_{c} \nabla \cdot \mathbf{w}\right) \delta_{i j} \\
+G\left(u_{i, j}+u_{j, i}-\frac{2}{3} u_{k, k} \delta_{i j}\right), \\
-P_{p}=c_{21} \nabla \cdot \mathbf{u}+\epsilon_{p} c_{22} \nabla \cdot \mathbf{v}+\epsilon_{c} c_{23} \nabla \cdot \mathbf{w}, \\
-P_{c}=c_{31} \nabla \cdot \mathbf{u}+\epsilon_{p} c_{23} \nabla \cdot \mathbf{v}+\epsilon_{c} c_{33} \nabla \cdot \mathbf{w},
\end{gathered}
$$

where $\delta_{i j}$ is Kronecker symbol. $B^{p}, B^{c}$ are Skempton's coefficients [20] for fluid pressures build up in pores and fractures, respectively. $K_{u}$ is bulk modulus of undrained porous solid, and $G$ is the rigidity of porous frame. The elastic tensor $c_{i j}$ is the inverse of a symmetric matrix $\left\{a_{i j}\right\}$.

In terms of the measurable quantities, the elements $a_{i j}$ are given by

$$
\begin{gathered}
a_{11}=\frac{1}{K}, \quad a_{12}=-\frac{\alpha^{(1)} K_{s}^{(1)}}{K_{s} K^{(1)}}, \\
a_{13}=-\frac{\alpha}{K}-a_{12}, \quad \alpha=1-\frac{K}{K_{s}},
\end{gathered}
$$




$$
\begin{gathered}
\alpha^{(1)}=1-\frac{K^{(1)}}{K_{s}^{(1)}}, \quad a_{22}=\frac{\left(1-\epsilon_{c}\right) \alpha^{(1)}}{B^{p} K^{(1)}}, \\
a_{23}=-\frac{\left(1-\epsilon_{c}\right) \alpha^{(1)}}{K^{(1)}}-a_{12}, \\
a_{33}=\frac{\epsilon_{c}}{K_{f}}+\frac{\epsilon_{s}+\epsilon_{p}}{K^{(1)}}-\frac{1-2 \alpha}{K}+2 a_{12},
\end{gathered}
$$

where $K, K^{(1)}\left(K_{s}, K_{s}^{(1)}\right)$ are the jacketed (unjacketed) bulk moduli of porous aggregate and solid matrix, respectively. $\alpha$ and $\alpha^{(1)}$ are the corresponding Biot-Willis parameters. $K_{f}$ is bulk modulus of saturating fluid. In terms of these coefficients, we have $K_{u}^{-1}=a_{11}-\left(a_{12}+a_{13}\right)^{2} /\left(a_{22}+a_{33}\right)$. The relations (3) are used to express the equations of motion, in terms of displacements and dilatations $\left(e_{s}=\nabla \cdot \mathbf{u}, e_{p}=\right.$ $\nabla \cdot \mathbf{v}$, and $\left.e_{c}=\nabla \cdot \mathbf{w}\right)$, as

$$
\begin{gathered}
\nabla\left[\left(K_{u}+\frac{1}{3} G\right) e_{s}-K_{u}\left(\epsilon_{p} B^{p} e_{p}+\epsilon_{c} B^{c} e_{c}\right)\right]+G \nabla^{2} \mathbf{u} \\
=\epsilon_{s} \rho_{s} \ddot{\mathbf{u}}+\rho_{12} \ddot{\mathbf{v}}+\rho_{13} \ddot{\mathbf{w}}-b_{12} \dot{\mathbf{v}}-b_{13} \dot{\mathbf{w}}, \\
\epsilon_{p} \nabla\left[c_{21} e_{s}+\epsilon_{p} c_{22} e_{p}+\epsilon_{c} c_{23} e_{c}\right] \\
=\epsilon_{p} \rho_{f} \ddot{\mathbf{u}}+\rho_{22} \ddot{\mathbf{v}}+\rho_{23} \ddot{\mathbf{w}}+\left(b_{12}+b_{23}\right) \dot{\mathbf{v}}-b_{23} \dot{\mathbf{w}}, \\
\epsilon_{c} \nabla\left[c_{31} e_{s}+\epsilon_{p} c_{32} e_{p}+\epsilon_{c} c_{33} e_{c}\right] \\
=\epsilon_{c} \rho_{f} \ddot{\mathbf{u}}+\rho_{23} \ddot{\mathbf{v}}+\rho_{33} \ddot{\mathbf{w}}-b_{23} \dot{\mathbf{v}}+\left(b_{13}+b_{23}\right) \dot{\mathbf{w}} .
\end{gathered}
$$

2.1. Viscoelastic Porous Frame. A poroviscoelastic solid saturated with viscous fluid represents a realistic homogeneous model for sedimentary or reservoir rocks. Biot-Stoll model [21] is an important mathematical model that takes into account both intergranular losses in solid frame and viscous losses in interstitial fluid. This is a very useful model to study the propagation of attenuated waves in marine sediments [22]. Sharma and Gogna [23] considered this model to study the reflection of attenuated body waves at its plane boundary. In the present problem, following Stoll [22], viscoelastic response of skeletal frame is defined by the complex transforms $K_{s}\left(1-\imath Q_{b}^{-1}\right), K_{s}^{(1)}\left(1-\imath Q_{b}^{-1}\right)$ and $G\left(1-\imath Q_{r}^{-1}\right)$ of its elastic moduli (i.e., $K_{s}, K_{s}^{(1)}$, and $G$ ). Values of quality factors are further related to $\log$ decrement parameters $\left(\delta_{G}, \delta_{E}\right)$ for rigidity $(G)$ and Young's modulus $(E)$ of drained porous frame. The relations, given by

$$
Q_{r}=\frac{\pi}{\delta_{G}}, \quad \frac{Q_{r}}{Q_{b}} \approx 5 \frac{\delta_{E}}{\delta_{G}}-4,
$$

define the attenuation from skeletal frame with the values of $\delta_{G}$ and $\delta_{E} / \delta_{G}$.

\section{General Solution: Wave Potentials}

Through the usual Helmholtz decomposition of a vector, the displacement vectors in three homogeneous isotropic constituents of porous aggregate are written as

$$
\begin{array}{cc}
\mathbf{u}=\nabla \phi+\nabla \times \mathbf{S}, & \nabla \cdot \mathbf{S}=0 \\
\mathbf{v}=\nabla \psi+\nabla \times \mathbf{F}_{\mathbf{p}}, & \nabla \cdot \mathbf{F}_{\mathbf{p}}=0 ; \\
\mathbf{w}=\nabla \xi+\nabla \times \mathbf{F}_{\mathbf{c}}, & \nabla \cdot \mathbf{F}_{\mathbf{c}}=0 .
\end{array}
$$

Using the above potentials, the system (5) contains thirdorder differential equations in the form $\nabla a+\nabla \times \mathbf{A}=\mathbf{B}=$ $0, \nabla \cdot \mathbf{A}=0$, involving scalar potentials $a$ and vector potentials A. In particular, we can write ([24], pp. 52) $a=\nabla \cdot \mathbf{P}$ and $\mathbf{A}=\nabla \times \mathbf{P}$, for some vector function $\mathbf{P}$ derived from the integration of $\mathbf{B}$. In the present case, $\mathbf{B}=0$ yields $\mathbf{P}=0$, and hence we get $a=0$ and $\mathbf{B}=0$. This provides us two systems of (second-order) differential equations, one in scalar potentials and the other corresponding to vector potentials. Finally, in terms of displacement potentials $\left(\phi, \psi, \xi ; \mathbf{S}, \mathbf{F}_{\mathbf{p}}, \mathbf{F}_{\mathbf{c}}\right)$, the system of (5) is resolved in two subsystems as follows:

$$
\begin{gathered}
\left(K_{u}+\frac{4}{3} G\right) \nabla^{2} \phi-\epsilon_{p} K_{u} B^{p} \nabla^{2} \psi-\epsilon_{c} K_{u} B^{c} \nabla^{2} \xi \\
=\epsilon_{s} \rho_{s} \ddot{\phi}+\left(\rho_{12} \ddot{\psi}-b_{12} \dot{\psi}\right)+\left(\rho_{13} \ddot{\xi}-b_{13} \dot{\xi}\right) \\
\epsilon_{p}\left(c_{21} \nabla^{2} \phi+\epsilon_{p} c_{22} \nabla^{2} \psi+\epsilon_{c} c_{23} \nabla^{2} \xi\right) \\
=\epsilon_{p} \rho_{f} \ddot{\phi}+\left[\rho_{22} \ddot{\psi}-\left(b_{12}+b_{23}\right) \dot{\psi}\right]+\left(\rho_{23} \ddot{\xi}-b_{23} \dot{\xi}\right), \\
\epsilon_{c}\left(c_{31} \nabla^{2} \phi+\epsilon_{p} c_{32} \nabla^{2} \psi+\epsilon_{c} c_{33} \nabla^{2} \xi\right) \\
=\epsilon_{c} \rho_{f} \ddot{\phi}+\left(\rho_{23} \ddot{\psi}-b_{23} \dot{\psi}\right)+\left[\rho_{33} \ddot{\xi}+\left(b_{13}+b_{23}\right) \dot{\xi}\right] \\
G \nabla^{2} \mathbf{S}=\epsilon_{s} \rho_{s} \ddot{\mathbf{S}}+\left(\rho_{12} \ddot{\mathbf{F}_{\mathbf{p}}}-b_{12} \dot{\mathbf{F}_{\mathbf{p}}}\right)+\left(\rho_{13} \ddot{\mathbf{F}_{\mathbf{c}}}-b_{13} \dot{\mathbf{F}_{\mathbf{c}}}\right) \\
0=\epsilon_{p} \rho_{f} \ddot{\mathbf{S}}+\rho_{22} \ddot{\mathbf{F}}_{\mathbf{p}}+\left(b_{12}+b_{23}\right) \dot{\mathbf{F}}_{\mathbf{p}}+\rho_{23} \ddot{\mathbf{F}}_{\mathbf{c}}-b_{23} \dot{\mathbf{F}_{\mathbf{c}}} \\
0=\epsilon_{c} \rho_{f} \ddot{\mathbf{S}}+\rho_{23} \ddot{\mathbf{F}_{\mathbf{p}}}-b_{23} \dot{\mathbf{F}}_{\mathbf{p}}+\rho_{33} \ddot{\mathbf{F}_{\mathbf{c}}}+\left(b_{13}+b_{23}\right) \dot{\mathbf{F}_{\mathbf{c}}}
\end{gathered}
$$

For time harmonic $\left(\sim e^{-\imath \omega t}\right)$ potentials $(\phi, \psi, \xi)$ to represent harmonic waves of angular frequency $\omega$, the system (8) transforms to

$$
\begin{gathered}
{\left[\left(K_{u}+\frac{4}{3} G\right) \nabla^{2}+\epsilon_{s} \rho_{s} \omega^{2}\right] \phi-\left(\epsilon_{p} K_{u} B^{p} \nabla^{2}+r_{12} \omega^{2}\right) \psi} \\
-\left(\epsilon_{c} K_{u} B^{c} \nabla^{2}+r_{13} \omega^{2}\right) \xi=0, \\
\left(\epsilon_{p} c_{21} \nabla^{2}+\epsilon_{p} \rho_{f} \omega^{2}\right) \phi+\left(\epsilon_{p}^{2} c_{22} \nabla^{2}+r_{22} \omega^{2}\right) \psi \\
+\left(\epsilon_{p} \epsilon_{c} \mathcal{c}_{23} \nabla^{2}+r_{23} \omega^{2}\right) \xi=0, \\
\left(\epsilon_{c} \mathcal{c}_{31} \nabla^{2}+\epsilon_{c} \rho_{f} \omega^{2}\right) \phi+\left(\epsilon_{p} \epsilon_{c} \mathcal{c}_{23} \nabla^{2}+r_{23} \omega^{2}\right) \psi \\
+\left(\epsilon_{c}^{2} c_{33} \nabla^{2}+r_{33} \omega^{2}\right) \xi=0,
\end{gathered}
$$


where $r_{22}=\rho_{22}+(l / \omega)\left(b_{12}+b_{23}\right), r_{33}=\rho_{33}+(l / \omega)\left(b_{13}+b_{23}\right)$, and $r_{i j}=\rho_{i j}-(\imath / \omega) b_{i j}(i<j=1,2,3)$.

The equations (11) are solved into two relations, given by

$$
\begin{aligned}
& \left(A_{1} \nabla^{4}+\omega^{2} B_{1} \nabla^{2}+\omega^{4} C_{1}\right) \psi=\left(A_{2} \nabla^{4}+\omega^{2} B_{2} \nabla^{2}+\omega^{4} C_{2}\right) \phi, \\
& \left(A_{1} \nabla^{4}+\omega^{2} B_{1} \nabla^{2}+\omega^{4} C_{1}\right) \xi=\left(A_{3} \nabla^{4}+\omega^{2} B_{3} \nabla^{2}+\omega^{4} C_{3}\right) \phi .
\end{aligned}
$$

Using these relations in (10), we obtain

$$
\left[A \nabla^{6}+\omega^{2} B \nabla^{4}+\omega^{4} C \nabla^{2}+\omega^{6} D\right] \phi=0,
$$

where

$$
\begin{gathered}
\left(\begin{array}{c}
A \\
B \\
C \\
D
\end{array}\right)=\left[\begin{array}{ccc}
A_{1} & A_{2} & A_{3} \\
B_{1} & B_{2} & B_{3} \\
C_{1} & C_{2} & C_{3} \\
0 & 0 & 0
\end{array}\right]\left(\begin{array}{c}
K_{u}+\frac{4 G}{3} \\
-\epsilon_{p} K_{u} B^{p} \\
-\epsilon_{c} K_{u} B^{c}
\end{array}\right) \\
+\left[\begin{array}{ccc}
0 & 0 & 0 \\
A_{1} & A_{2} & A_{3} \\
B_{1} & B_{2} & B_{3} \\
C_{1} & C_{2} & C_{3}
\end{array}\right]\left(\begin{array}{c}
\epsilon_{s} \rho_{s} \\
r_{12} \\
r_{13}
\end{array}\right) ; \\
A_{1}=\delta_{p}^{2} \delta_{c}^{2}\left(c_{22} c_{33}-c_{23}^{2}\right), \\
B_{1}=\delta_{p}^{2} c_{22} r_{33}+\delta_{c}^{2} c_{33} r_{22}-2 \epsilon_{p} \epsilon_{c} c_{23} r_{23}, \\
C_{1}=r_{22} r_{33}-r_{23}^{2}, \quad A_{2}=\epsilon_{p} \delta_{c}^{2}\left(c_{23} c_{13}-c_{12} c_{33}\right), \\
B_{2}=\epsilon_{p} \delta_{c}^{2} \rho_{f}\left(c_{23}-c_{33}\right)+\epsilon_{c} c_{13} r_{23}-\epsilon_{p} c_{12} r_{33}, \\
C_{2}=\rho_{f}\left(\epsilon_{c} r_{23}-\epsilon_{p} r_{33}\right), \quad A_{3}=\delta_{p}^{2} \epsilon_{c}\left(c_{12} c_{23}-c_{13} c_{22}\right), \\
B_{3}=\delta_{p}^{2} \epsilon_{c} \rho_{f}\left(c_{23}-c_{22}\right)+\epsilon_{p} c_{12} r_{23}-\epsilon_{c} c_{13} r_{22}, \\
C_{3}=\rho_{f}\left(\epsilon_{p} r_{23}-\epsilon_{c} r_{22}\right) .
\end{gathered}
$$

The differential equation (13) is decomposed to satisfy three Helmholtz equations, given by

$$
\left(\nabla^{2}+\frac{\omega^{2}}{V_{i}^{2}}\right) \phi_{i}=0 \quad(i=1,2,3) .
$$

The velocities $\left(V_{i}\right)$ are derived from the roots of a cubic equation in $V^{2}$, given by

$$
D V^{6}-C V^{4}+B V^{2}-A=0
$$

and are sorted in the descending order of their real parts. The system (15) thus implies the existence of three dilatational waves (named $P_{1}, P_{2}, P_{3}$ ) propagating with phase velocities $V_{i}(i=1,2,3)$ and identified with the corresponding scalar potentials $\phi_{i}$.

In the considered linear porous medium, the general representation of potential function for aggregate dilatation is expressed as

$$
\phi=\phi_{1}+\phi_{2}+\phi_{3}
$$

which, on using in relations (12), yields

$$
\psi=\mu_{1} \phi_{1}+\mu_{2} \phi_{2}+\mu_{3} \phi_{3}, \quad \xi=v_{1} \phi_{1}+v_{2} \phi_{2}+v_{3} \phi_{3},
$$

where

$$
\mu_{j}=\frac{A_{2}-B_{2} V_{j}^{2}+C_{2} V_{j}^{4}}{A_{1}-B_{1} V_{j}^{2}+C_{1} V_{j}^{4}}
$$

$$
v_{j}=\frac{A_{3}-B_{3} V_{j}^{2}+C_{3} V_{j}^{4}}{A_{1}-B_{1} V_{j}^{2}+C_{1} V_{j}^{4}} \quad(j=1,2,3) \text {. }
$$

Similar to scalar potentials considered above, vector potentials $\left(\mathbf{S}, \mathbf{F}_{\mathbf{p}}, \mathbf{F}_{\mathbf{c}}\right)$ are also considered as time harmonic with frequency $\omega$. Then, for the time harmonic dependence, given by $\left(\sim e^{-i \omega t}\right)$, the system of three equations (9) is resolved into another set of three equations, given by

$$
\begin{gathered}
\left(G \nabla^{2}+\epsilon_{s} \rho_{s} \omega^{2}\right) \mathbf{S}=r_{12} \omega^{2} \mathbf{F}_{\mathbf{p}}+r_{13} \omega^{2} \mathbf{F}_{\mathbf{c}} \\
\epsilon_{p} \rho_{f} \mathbf{S}+r_{12} \mathbf{F}_{\mathbf{p}}+r_{23} \mathbf{F}_{\mathbf{c}}=0, \quad \epsilon_{c} \rho_{f} \mathbf{S}+r_{23} \mathbf{F}_{\mathbf{p}}+r_{33} \mathbf{F}_{\mathbf{c}}=0 .
\end{gathered}
$$

Solving the above relations, we get a Helmholtz equation, given by

$$
\begin{gathered}
\left(\nabla^{2}+\frac{\omega^{2}}{V_{4}^{2}}\right) \mathbf{S}=0 ; \quad V_{4}^{2}=\frac{G}{\left(\epsilon_{s} \rho_{s}-\mu_{4} r_{12}-\nu_{4} r_{13}\right)}, \\
\mu_{4}=\frac{C_{2}}{C_{1}}, \quad v_{4}=\frac{C_{3}}{C_{1}} ;
\end{gathered}
$$

which defines the existence of a shear (or $S$ ) wave propagating with velocity $V_{4}$. The parameters $\mu_{4}$ and $\nu_{4}$ also relate the vector potentials as $\left(\mathbf{F}_{\mathbf{p}}, \mathbf{F}_{\mathbf{c}}\right)=\left(\mu_{4}, v_{4}\right) \mathbf{S}$.

\section{Velocity and Attenuation}

In the previous section, the existence of four (three dilatational and one shear) waves has been explained. The complex velocities $V_{1}, V_{2}, V_{3}$ of three dilatational waves, named $P_{1}, P_{2}, P_{3}$, respectively, are obtained from the roots of the cubic equation (16). The propagation of lone shear (or $S$ ) wave is represented through the complex velocity $V_{4}$, given by (21). The complex velocity, say $V_{k}$, of any of these four waves is resolved to define the corresponding phase (or propagation) velocity $\left(v_{k}\right)$ and attenuation quality factor $\left(Q_{k}\right)$ as follows:

$$
v_{k}=\frac{\left|V_{k}\right|^{2}}{\mathfrak{R}\left(V_{k}\right)}, \quad Q_{k}^{-1}=-\frac{\mathfrak{I}\left(V_{k}^{2}\right)}{\mathfrak{R}\left(V_{k}^{2}\right)} .
$$

\section{Numerical Example}

Berea sandstone is considered as a physical model of the porous medium. Following Stoll [22], the values of various parameters are chosen for sandstone with water in its pores and fractures. The skeletal frame of sandstone consists of solid grains with bulk modulus $K_{s}=38 \mathrm{GPa}$, rigidity modulus 


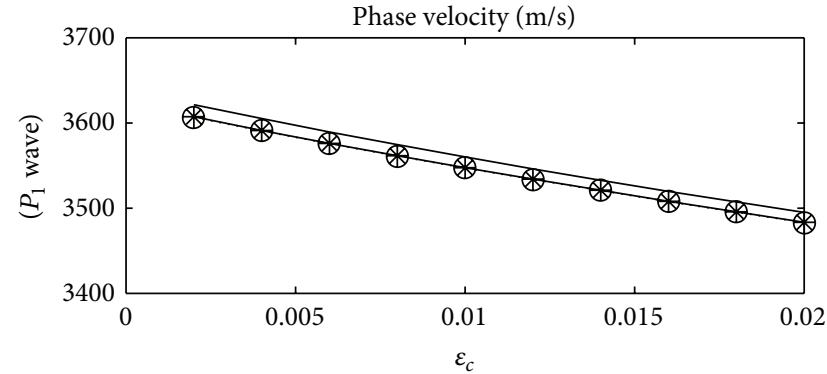

(a)

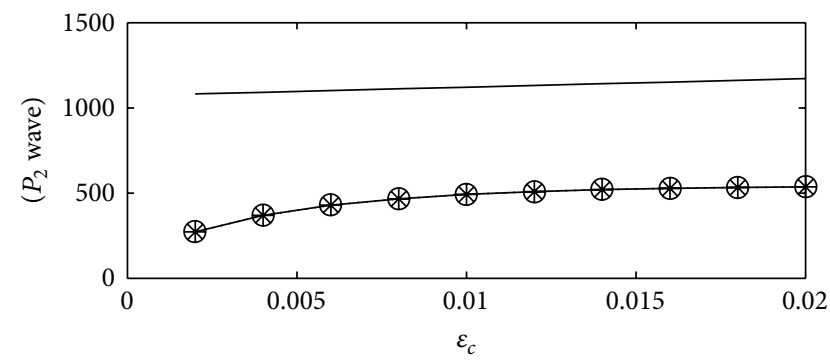

(c)

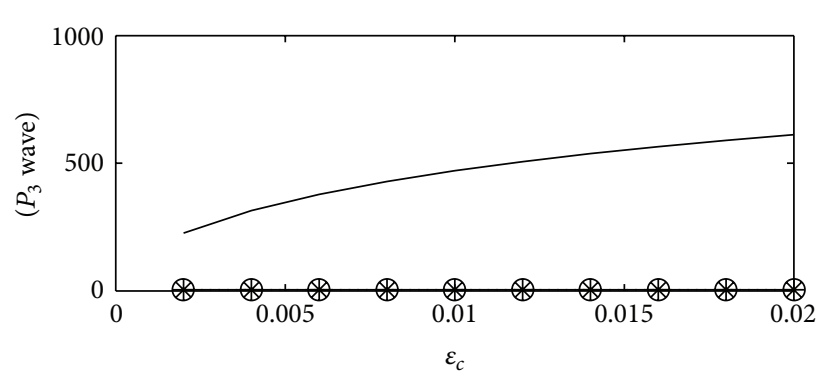

(e)

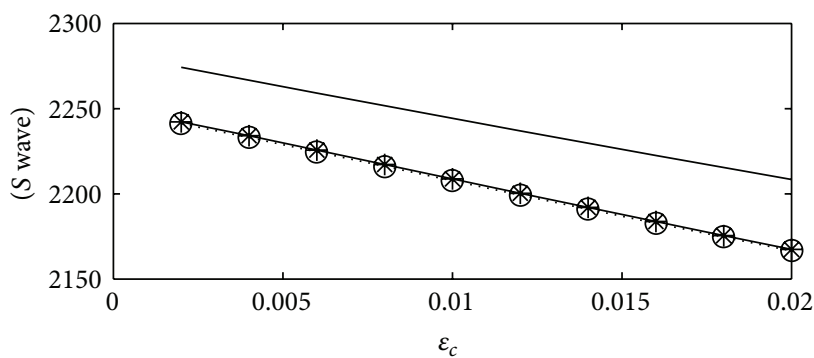

- Nondissipative

$\bigcirc$. Viscous fluid

* Anelastic solid + viscous fluid

(g)

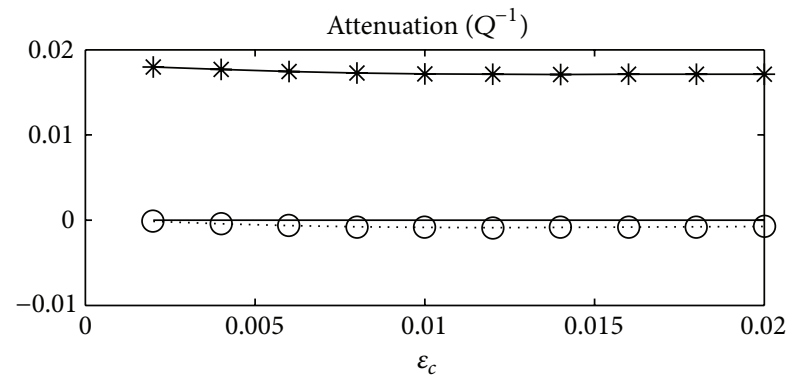

(b)

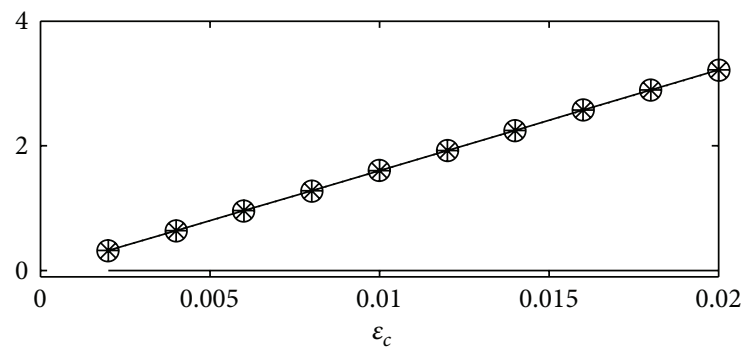

(d)

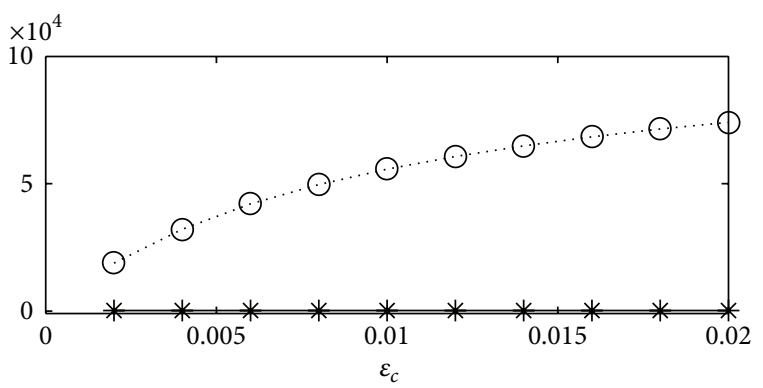

(f)

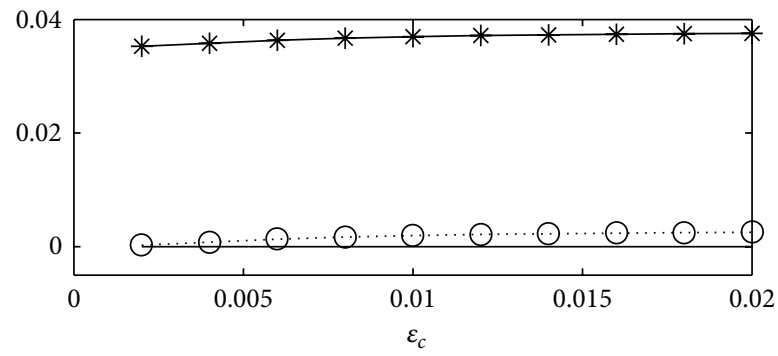

- Nondissipative

. $\odot$. Viscous fluid

* Anelastic solid + viscous fluid

FIGURE 1: Variations of wave velocities and attenuation with fracture porosity in Berea sandstone; effect of dissipation from fluid viscosity or frame anelasticity (viscous fluid: $\eta=0.001$ poise; pore permeability: $\chi_{11}=10^{-16} \mathrm{~m}^{2}$; fracture permeability: $\chi_{22}=10^{-12} \mathrm{~m}^{2}$; anelastic frame: $\delta_{G}=0.11, \delta_{E} / \delta_{G}=0.9$; wave frequency: $1000 \mathrm{~Hz}$ ). 


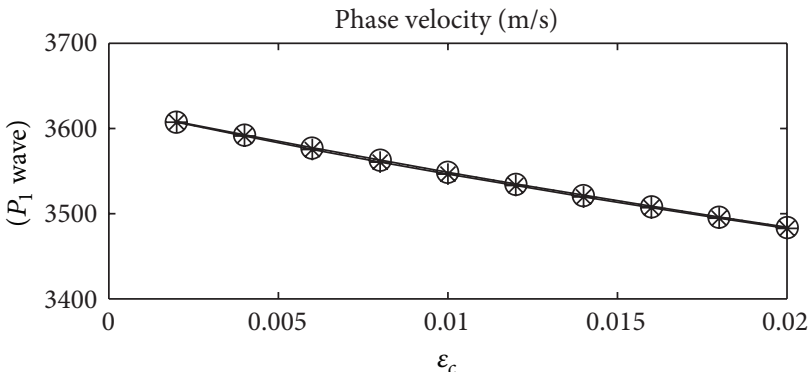

(a)

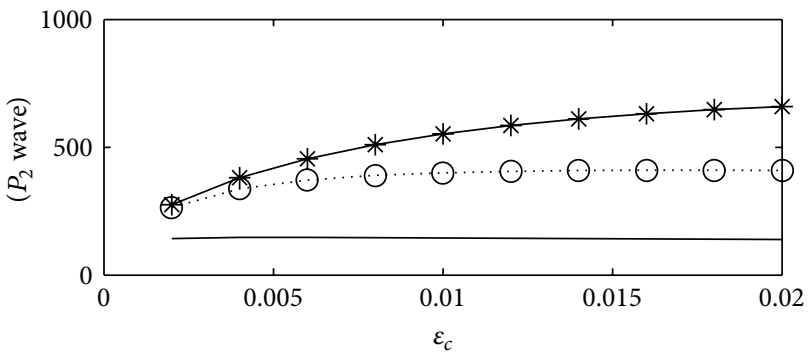

(c)

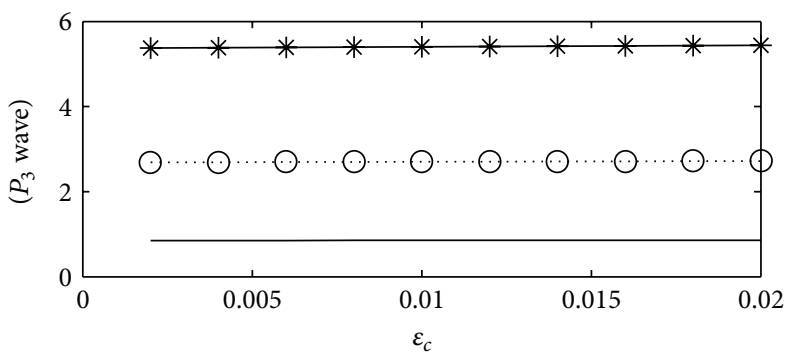

(e)

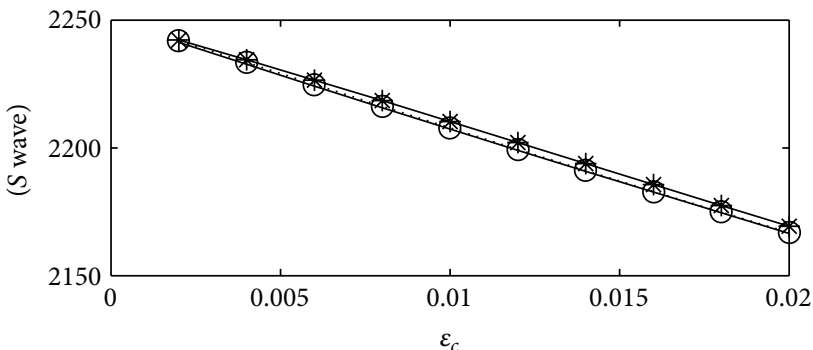

$-50 \mathrm{~Hz}$

๑) $500 \mathrm{~Hz}$

* $2000 \mathrm{~Hz}$

(g)

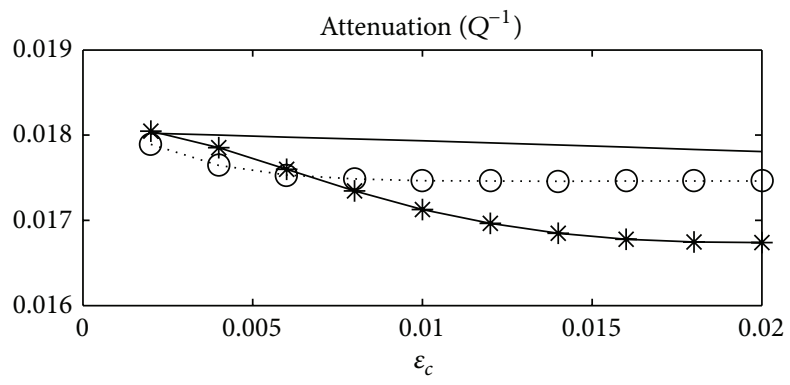

(b)

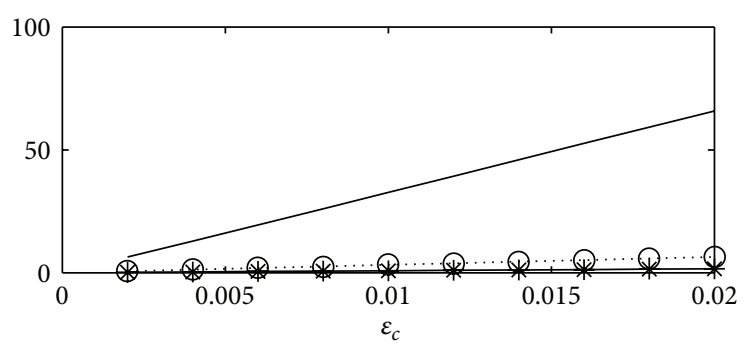

(d)

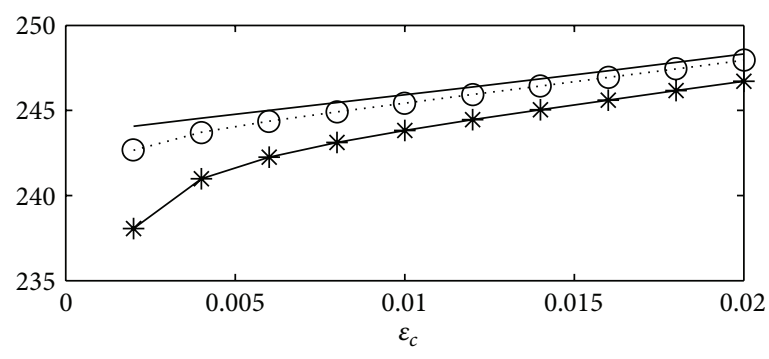

(f)

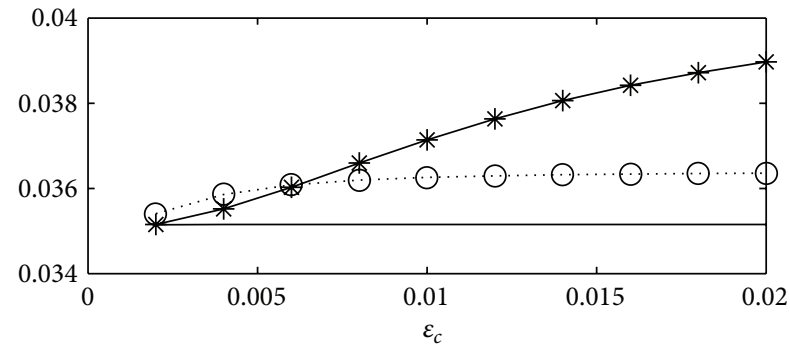

$50 \mathrm{~Hz}$

. $5.500 \mathrm{~Hz}$

* $2000 \mathrm{~Hz}$

(h)

FIGURE 2: Variations of wave velocities and attenuation with fracture porosity in Berea sandstone; effect of wave frequency (viscous fluid: $\eta=0.001$ poise; pore permeability: $\chi_{11}=10^{-16} \mathrm{~m}^{2}$; fracture permeability: $\chi_{22}=10^{-12} \mathrm{~m}^{2}$; anelastic frame: $\delta_{G}=0.11, \delta_{E} / \delta_{G}=0.9$ ). 


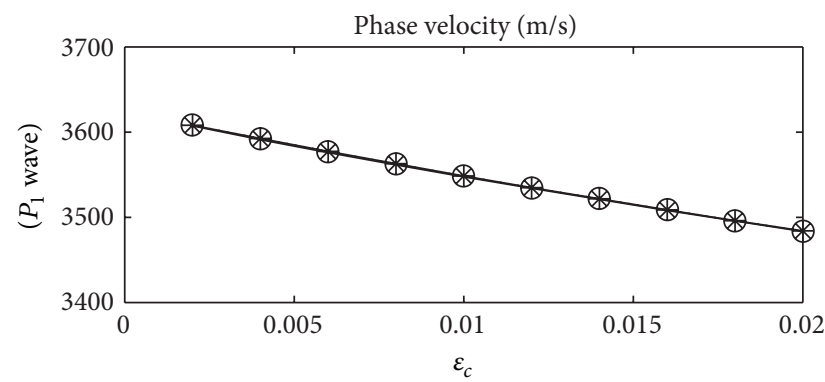

(a)

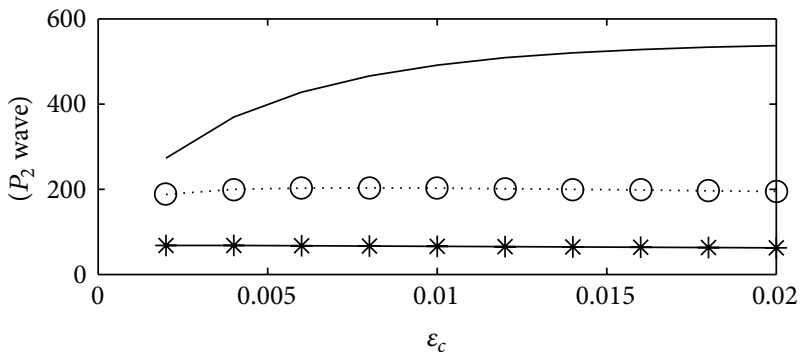

(c)

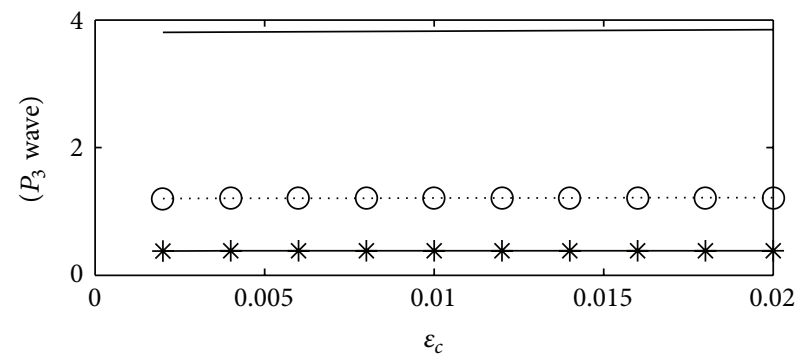

(e)

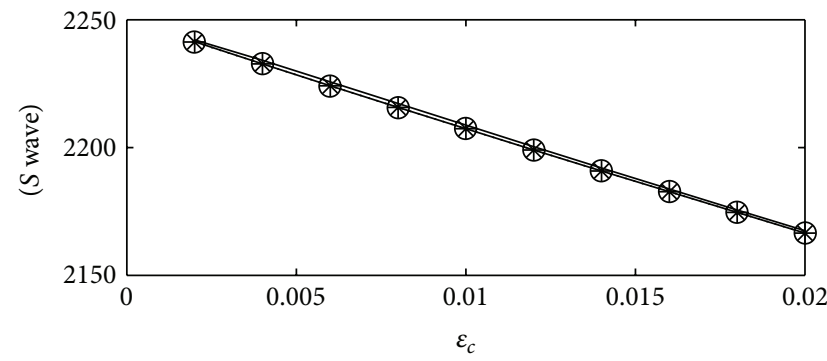

$\eta=0.001$ poise

○. $\eta=0.01$ poise

* $\eta=0.1$ poise

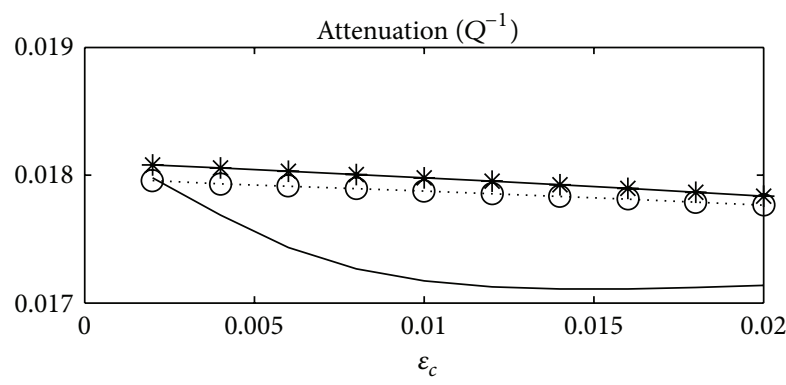

(b)

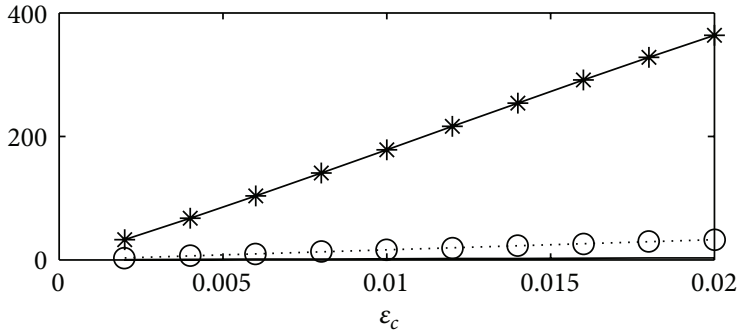

(d)

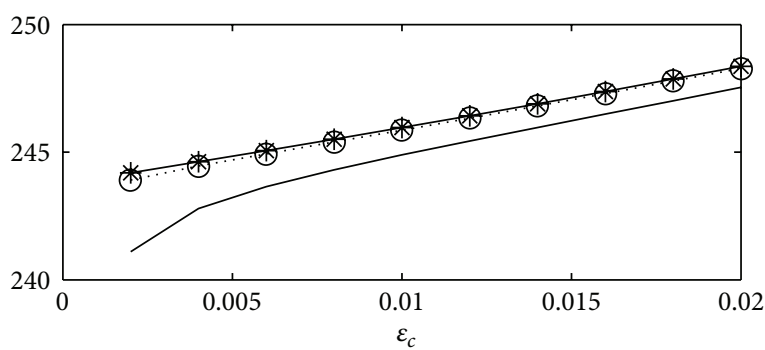

(f)

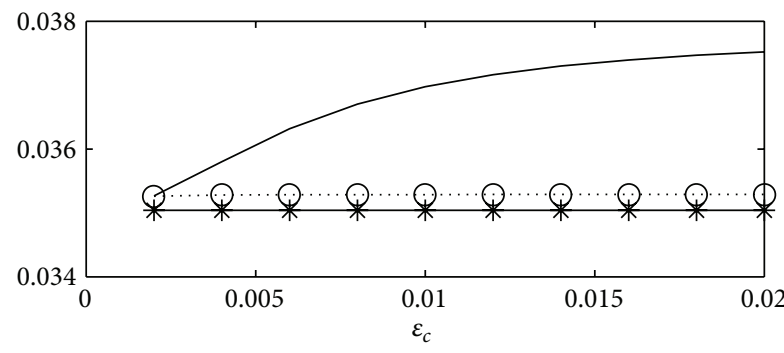

$$
\begin{aligned}
\eta & =0.001 \text { poise } \\
\bigcirc \cdot \eta & =0.01 \text { poise } \\
* \quad \eta & =0.1 \text { poise }
\end{aligned}
$$

(h)

FIGURE 3: Variations of wave velocities and attenuation with fracture porosity in Berea sandstone; effect of viscosity of interstitial fluid (pore permeability: $\chi_{11}=10^{-16} \mathrm{~m}^{2}$; fracture permeability: $\chi_{22}=10^{-12} \mathrm{~m}^{2}$; anelastic frame: $\delta_{G}=0.11, \delta_{E} / \delta_{G}=0.9$; wave frequency: $1000 \mathrm{~Hz}$ ). 
$G_{s}=44 \mathrm{GPa}$, and density $\rho_{s}=2650 \mathrm{~kg} / \mathrm{m}^{3}$. It supports the porosity $f=\epsilon_{p}+\epsilon_{c}$, where volume fraction of pores is fixed as $\epsilon_{p}=0.2$ and volume fraction of fractures is varied up to 0.02 . Both pores and fractures are filled with water of bulk modulus $K_{f}=2.3 \mathrm{GPa}$ and density $\rho_{f}=1000 \mathrm{~kg} / \mathrm{m}^{3}$. The rigidity modulus of the consolidated porous aggregate is calculated from $G_{s}$ as $G=(1-f) G_{s} /\left(1+c_{s} f\right)$ with consolidation parameter $c_{s}=10$ [18]. The bulk modulus of the consolidated porous aggregate is $K=6 \mathrm{GPa}$. Unjacketed bulk moduli are $K^{(1)}=10 \mathrm{GPa}$ and $K_{s}^{(1)}=K_{s}$. The dynamic viscosity of water $\eta=0.001$ poise makes the double porosity solid a dissipative one, which supports the attenuated propagation of waves. The permeability of the solid matrix to conduct the flow of fluid in pores and fractures is represented as $\chi_{11}=10^{-16} \mathrm{~m}^{2}, \chi_{12}=0$, and $\chi_{22}=10^{-12} \mathrm{~m}^{2}$. Moreover, the skeletal frame is considered viscoelastic solid with complex values for elastic constants for bulk moduli and rigidity modulus. Stoll [22] has estimated the log decrement values $\delta_{G}=0.11$ and $\delta_{E} / \delta_{G}=0.9$, for near elastic materials. These values are used in relations (22) to calculate the quality factors for elastic moduli of porous frame. Wave frequency restricted up to $2 \mathrm{kHz}$ ensures a low-frequency propagation. Skempton's coefficients for buildup of fluid pressures in pores and fractures are given by $B^{p}=0.6$ and $B^{c}=0.8$, respectively. For these numerical values of various parameters, the phase velocities $\left(v_{j}, j=1,2,3,4\right)$ and attenuation coefficients $\left(Q_{j}^{-1}, j=1,2,3,4\right)$ are calculated for the propagation of four waves in a double porosity medium. Variations of these velocities and attenuation coefficients with fracture porosity $\epsilon_{c}$ are plotted in Figures 1 to 4.

\section{Discussion of Numerical Results}

Figure 1 exhibits the variations of phase velocities and attenuations of four waves in double porosity medium (Berea sandstone) with the fracture porosity $\left(\epsilon_{c}\right)$ due to the presence of viscosity in saturating fluid and the anelasticity of skeletal frame. It is clear from the solid line curves that attenuation is absent when medium in nondissipative, that is, an elastic solid frame with inviscid fluid in pores and fractures. Velocities of all the waves decrease with the presence of dissipation, be it from viscous interstitial fluid or anelastic frame. The presence of attenuation comes from both, the viscous saturating fluid as well as anelastic solid frame. However, the presence of viscosity in fluid shows its effect mainly on slower $P$ waves. On the other hand, the attenuation in faster waves (i.e., $P_{1}$ and $S$ waves) comes mainly from anelasticity of solid frame. Velocities of these faster waves decrease with the increase of fracture porosity $\left(\epsilon_{c}\right)$. But the velocities of slower $P$ waves increase with the increase of $\epsilon_{c}$. Attenuation of slower $P$ waves and $S$ wave increase with the increase of $\epsilon_{c}$ but reverse may be case for fastest $\left(P_{1}\right)$ wave. However, the increase is much more in case of $P_{2}$ wave. In general, faster a wave is, the lesser its attenuation is. The strange behavior is noted for the attenuation of $P_{3}$ wave, which appears very strong with viscous fluid only. The reason is that the $P_{3}$ wave is the result of the presence of fracture porosity; therefore its extreme sensitivity to the changes in fracture properties may be expected. Note that, in case of anelastic or viscous dissipation, the velocity of this wave is negligible.

From the theoretical derivations, it is clear that complex velocities $\left(V_{j}, j=1,2,3,4\right)$ depend on the angular frequency. In Figure 2, variations of phase velocities and attenuations in Berea sandstone with the fracture porosity $\left(\epsilon_{c}\right)$ are exhibited for three values of frequency, that is, $50 \mathrm{~Hz}, 500 \mathrm{~Hz}$, and $2000 \mathrm{~Hz}$. From the first column plots in this figure, it is observed that frequency has no effect on the velocity of $P_{1}$ wave. The $S$ wave may propagate a little faster at high frequency. The slower $P$ waves are very sensitive to the wave frequency and propagate significantly faster with an increase in frequency. However, the velocity increase with frequency increases with $\epsilon_{c}$ in case of $P_{2}$ wave only. The attenuation of each of the three $P$ waves decreases slightly with the increase of frequency. It is only the $S$ wave, which attenuates more at high frequency. Notable point is that the effect of frequency is coupled with the presence of fractures. For example, the effect of frequency on each wave (except $P_{3}$ wave) is almost absent at smaller $\epsilon_{c}$ and it becomes more significant with increasing $\epsilon_{c}$.

With the presence of viscous fluid in pores and fracture, it becomes important to observe how the amount of this viscosity affects the velocities and attenuation of the four waves in a double porosity medium. Figure 3 illustrates this effect on the propagation and attenuation of four waves in Berea's sandstone. In general, the phase velocity of each wave decreases with an increase of viscosity. However, this decrease is negligible for $P_{1}$ wave, very slight for $S$ wave, and very significant in case of two slower $P$ waves. The velocity of $P_{2}$ wave appears, in general, to be sensitive to the extent of fractures in the porous medium, but in case of large fluid viscosity it loses this sensitivity. The reason may be the stronger glubeing effect of highly viscous fluid occupying a comparatively larger volume in fractures. The attenuation of each of the three $P$ waves increases with the increase of fluid viscosity. But opposite is the case with $S$ wave, where the attenuation decreases with increase in $\eta$. Sensitivity of attenuation to viscosity change, in general, increases with the increase in $\epsilon_{c}$.

Permeability of fracture network is important in conducting the flow of fluid in a saturated porous solid. In Figure 4 , the effect of fracture permeability $\left(\chi_{22}\right)$ is exhibited on the variations of velocities and attenuations of four waves in Berea's sandstone. The effect of this permeability on velocities is nearly similar to that of fluid viscosity. For example, its effect is negligible of $P_{1}$ wave, very slight on $S$ wave, and very significant of two slower $P$ waves. However, the velocities of these two waves increase with permeability, which is opposite to the effect of fluid viscosity. On the other hand, the effect of permeability on attenuation is different on different waves. In general, the increase in fracture permeability decreases the attenuation of all the three $P$ waves but the attenuation of $S$ wave increases with the increase in $\chi_{22}$. Sensitivity of attenuation to 


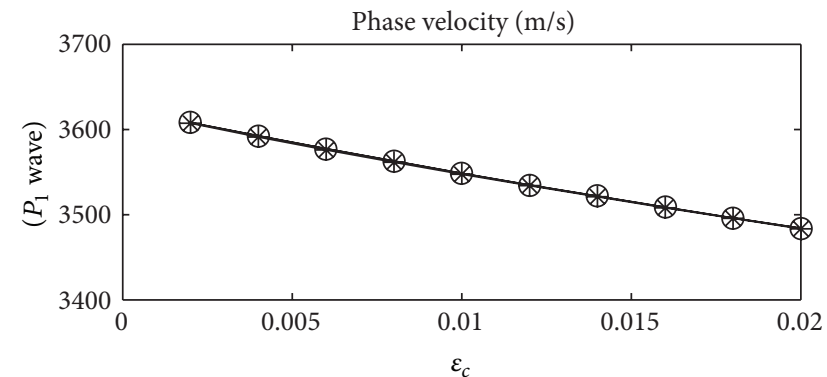

(a)

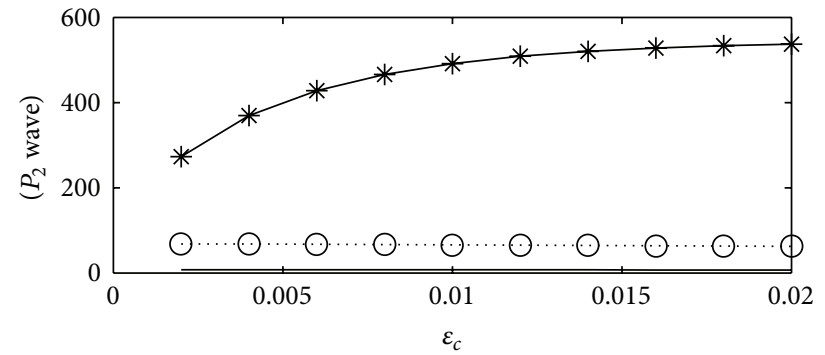

(c)

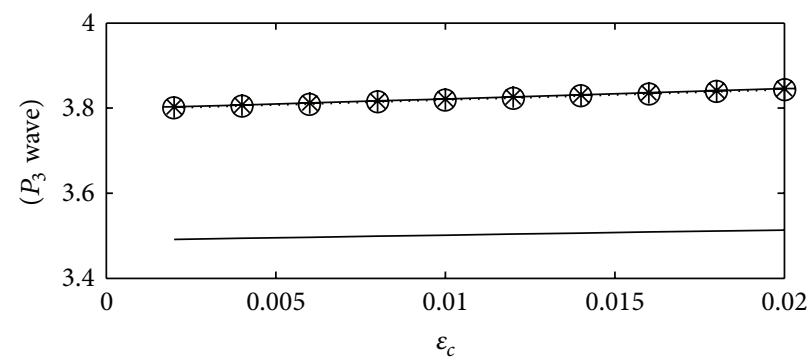

(e)

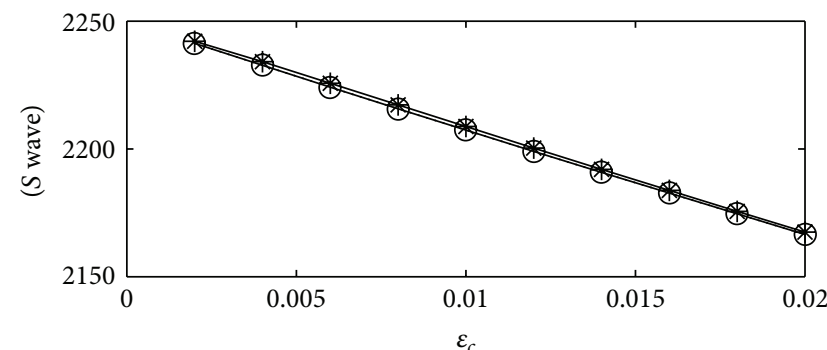

$\chi_{22}=10^{-16} \mathrm{~m}^{2}$

. $\chi_{22}=10^{-14} \mathrm{~m}^{2}$

* $\chi_{22}=10^{-12} \mathrm{~m}^{2}$

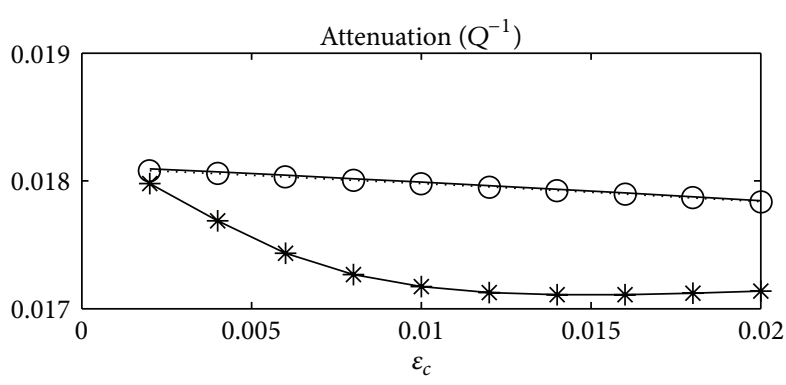

(b)

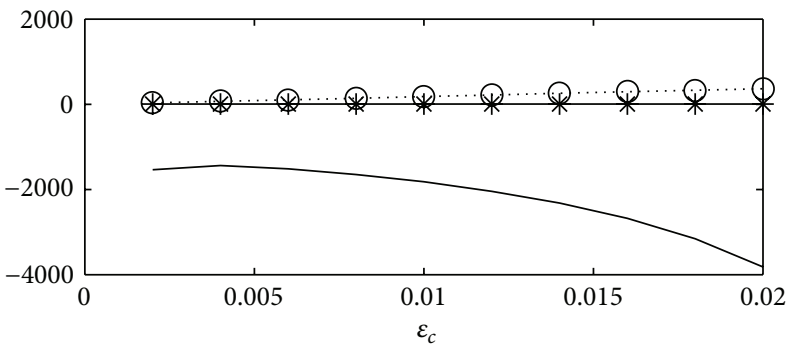

(d)

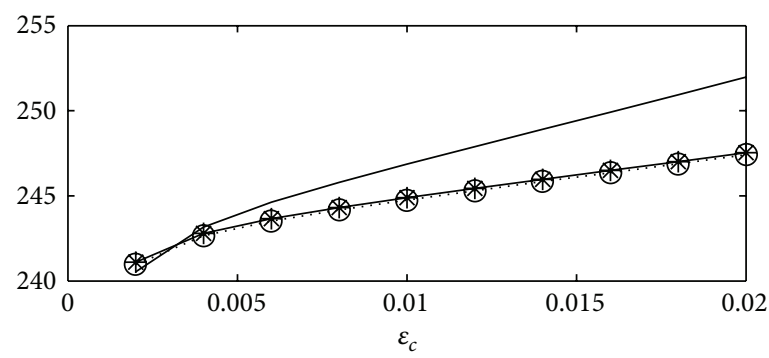

(f)

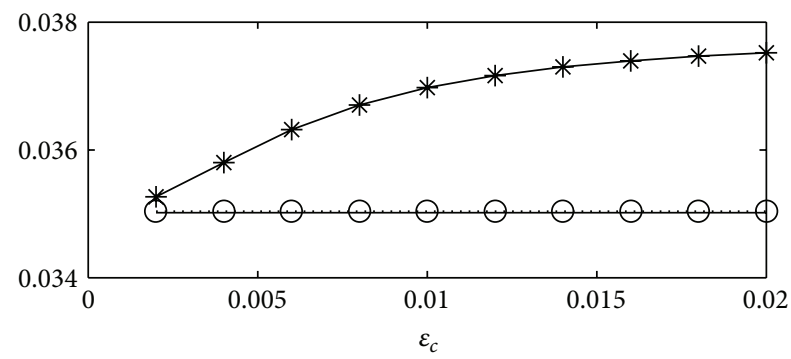

$-\chi_{22}=10^{-16} \mathrm{~m}^{2}$
$\odot \chi_{22}=10^{-14} \mathrm{~m}^{2}$
$* \chi_{22}=10^{-12} \mathrm{~m}^{2}$

(h)

FIGURE 4: Variations of wave velocities and attenuation with fracture porosity in Berea sandstone; effect of fracture permeability (viscous fluid: $\eta=0.001$ poise; pore permeability: $\chi_{11}=10^{-16} \mathrm{~m}^{2}$; anelastic frame: $\delta_{G}=0.11, \delta_{E} / \delta_{G}=0.9$; wave frequency: $1000 \mathrm{~Hz}$ ). 
fracture permeability increases with the increase in fracture porosity.

\section{Concluding Remarks}

The modeling and inversion procedures are used to interpret the measurable seismic quantities, like velocities and quality factors of attenuation, into the in situ properties of crustal rocks. The in-depth understanding of the relations between seismic properties and rock characteristics always helps in designing more effective mathematical models for seismic dynamism in the crust. It is well known in the phenomenology of earth materials that rocks are generally heterogeneous, porous, and often fractured or cracked. In situ, rock pores and cracks/fractures can contain oil, gas, or water. These fluid reservoirs are of great practical interest for science and economy in any society. Fracture or crack porosity may occupy very little volume but nevertheless has two very important effects on the reservoir properties. The first effect is that fractures/cracks drastically weaken the rock elastically, so that even a very small change in stress can lead to large changes in the fracture/crack apertures (and at the same time change the fracture strength for future changes). The second effect is that the fractures/cracks often introduce a high permeability pathway for the fluid to escape from the reservoir. This effect obviously is the key to reservoir analysis and the economics of fluid withdrawal.

\section{References}

[1] G. I. Barenblatt, I. P. Zheltov, and I. N. Kochina, "Basic concepts in the theory of seepage of homogeneous liquids in fissured rocks," Journal of Applied Mathematics and Mechanics, vol. 24, no. 5, pp. 1286-1303, 1960.

[2] J. E. Warren and P. J. Root, "The behavior of naturally fractured reservoirs," Society of Petroleum Engineers Journal, vol. 3, pp. 245-255, 1963.

[3] M. A. Biot, "Theory of propagation of elastic waves in a fluidsaturated porous solid. I. Low frequency range. II. Higher frequency range," Journal of the Acoustical Society of America, vol. 28, pp. 168-191, 1956.

[4] M. A. Biot, "Mechanics of deformation and acoustic propagation in porous media," Journal of Applied Physics, vol. 33, no. 4, pp. 1482-1498, 1962.

[5] M. A. Biot, "Generalized theory of acoustic propagation in porous dissipative media," Journal of the Acoustical Society of America, vol. 34, pp. 1254-1264, 1962.

[6] R. I. O'Connell and B. Budiansky, "Viscoelastic properties of fluid saturated cracked solids," Journal of Geophysical Research, vol. 82, pp. 5719-5735, 1977.

[7] G. M. Mavko and A. Nur, "Wave attenuation in partially saturated rocks," Geophysics, vol. 44, no. 2, pp. 161-178, 1979.

[8] G. Mavko and D. Jizba, "Estimating grain-scale fluid effects on velocity dispersion in rocks," Geophysics, vol. 56, no. 12, pp. 1940-1949, 1991.

[9] J. Dvorkin and A. Nur, "Dynamic poroelasticity: a unified model with the squirt and the Biot mechanisms," Geophysics, vol. 58, no. 4, pp. 524-533, 1993.

[10] L. Thomsen, "Elastic anisotropy due to aligned cracks in porous rock," Geophysical Prospecting, vol. 43, no. 6, pp. 805-829, 1995.
[11] M. D. Sharma, "Surface-wave propagation in a cracked poroelastic half-space lying under a uniform layer of fluid," Geophysical Journal International, vol. 127, no. 1, pp. 31-39, 1996.

[12] R. K. Wilson and E. C. Aifantis, "On the theory of consolidation with double porosity," International Journal of Engineering Science, vol. 20, pp. 1009-1035, 1982.

[13] R. K. Wilson and E. C. Aifantis, "A double porosity model for acoustic wave propagation in fractured-porous rock," International Journal of Engineering Science, vol. 22, no. 8-10, pp. 12091217, 1984.

[14] T. F. Cho, M. E. Plesha, and B. C. Haimson, "Continuum modelling of jointed porous rock," International Journal for Numerical \& Analytical Methods in Geomechanics, vol. 15, no. 5, pp. 333-353, 1991.

[15] M. Bai, D. Elsworth, and J. C. Roegiers, "Modeling of naturally fractured reservoirs using deformation dependent flow mechanism," International Journal of Rock Mechanics and Mining Sciences and, vol. 30, no. 7, pp. 1185-1191, 1993.

[16] J. G. Berryman and H. F. Wang, "The elastic coefficients of double-porosity models for fluid transport in jointed rock," Journal of Geophysical Research, vol. 100, no. 12, pp. 611-627, 1995.

[17] J. G. Berryman and H. F. Wang, "Elastic wave propagation and attenuation in a double-porosity dual-permeability medium," International Journal of Rock Mechanics and Mining Sciences, vol. 37, no. 1-2, pp. 63-78, 2000.

[18] S. R. Pride and J. G. Berryman, "Linear dynamics of doubleporosity dual-permeability materials. I. Governing equations and acoustic attenuation," Physical Review E, vol. 68, no. 3, Article ID 036603, 10 pages, 2003.

[19] S. R. Pride and J. G. Berryman, "Linear dynamics of doubleporosity dual-permeability materials. II. Fluid transport equations," Physical Review E, vol. 68, no. 3, Article ID 036604, 10 pages, 2003.

[20] A. W. Skempton, "The pore-pressure coefficients A and B," Geotechnique, vol. 4, pp. 143-147, 1954.

[21] R. D. Stoll and G. M. Bryan, "Wave attenuation in saturated sediments," Journal of the Acoustical Society of America, vol. 47, pp. 1440-1147, 1970.

[22] R. D. Stoll, "Marine sediment acoustics," Journal of the Acoustical Society of America, vol. 77, no. 5, pp. 1789-1799, 1985.

[23] M. D. Sharma and M. L. Gogna, "Seismic wave propagation in a viscoelastic porous solid saturated by viscous liquid," Pure and Applied Geophysics, vol. 135, no. 3, pp. 383-400, 1991.

[24] P. M. Morse and H. Feshback, Methods of Theoretical Physics, McGraw-Hill, New York, NY, USA, 1953. 

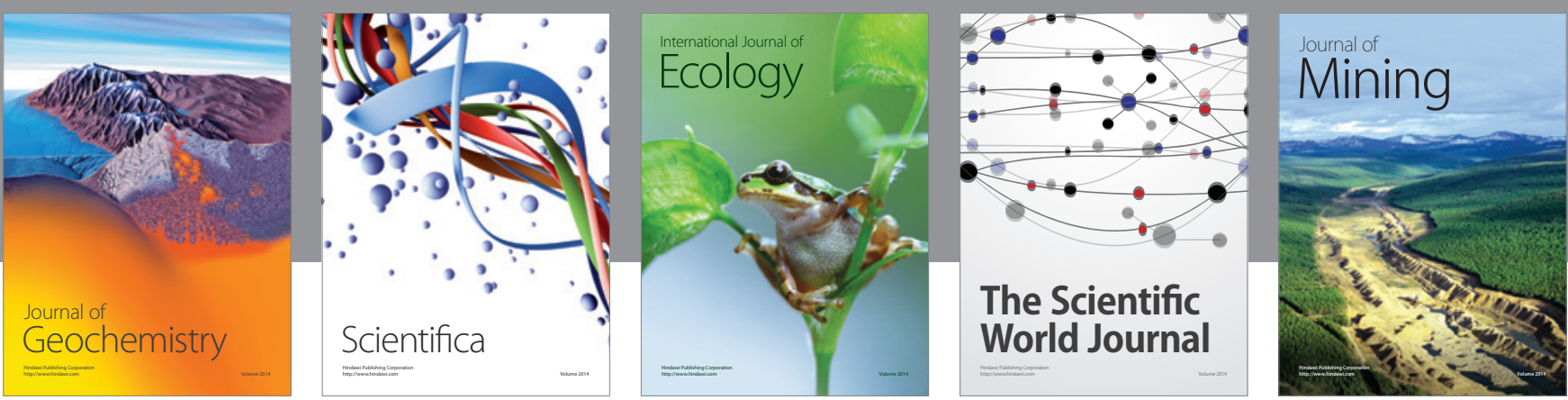

The Scientific World Journal
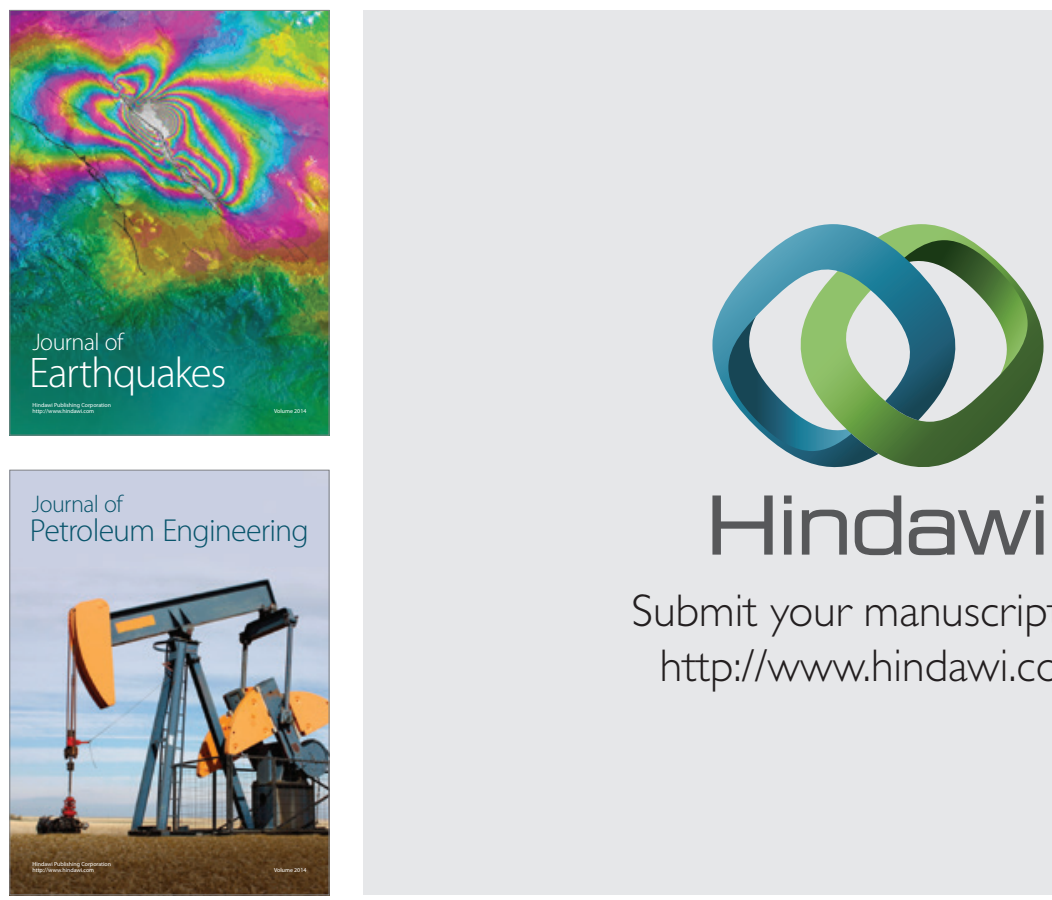

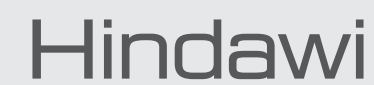

Submit your manuscripts at

http://www.hindawi.com
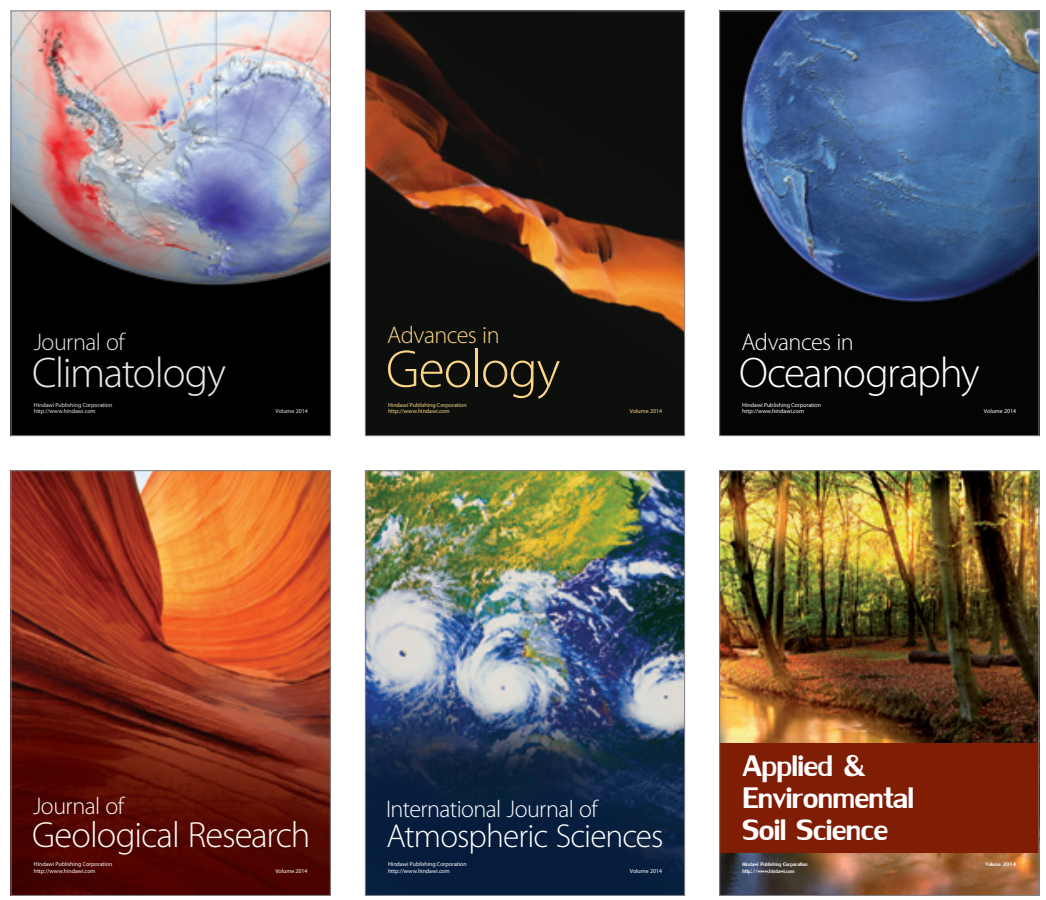
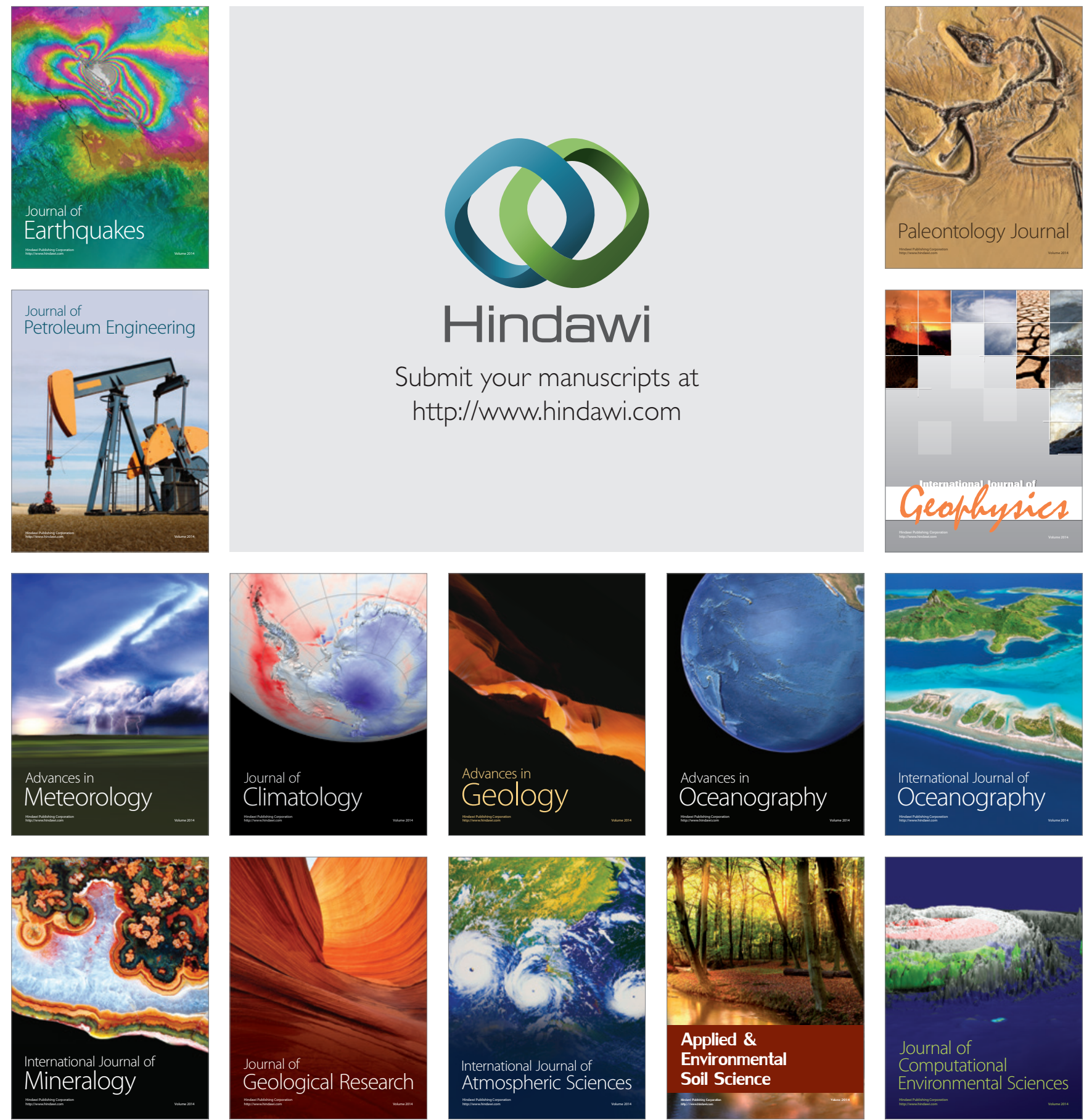